\title{
Graphene exfoliation in organic solvents and switching solubility in aqueous media with the aid of amphiphilic block copolymers
}

\author{
Theodosis Skaltsas ${ }^{a}$, Nikolaos Karousis ${ }^{a}$, Hui-Juan Yan ${ }^{b}$, Chun-Ru Wang ${ }^{b}$, Stergios Pispas*a and Nikos

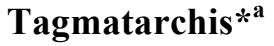

\author{
5 Received (in $X X X, X X X)$ Xth $X X X X X X X X X 20 X X$, Accepted $X$ th $X X X X X X X X X 20 X X$ \\ DOI: $10.1039 / b 000000 x$
}

\begin{abstract}
The successful exfoliation of graphite to graphene sheets in liquid phase via tip sonication was achieved. A number of solvents were examined for several time periods and it was found that $o$-dichlorobenzene (oDCB) and N-methyl-1,2-pyrolidone (NMP) are ideal solvents to exfoliate graphite and produce stable 10 dispersions of graphene. The exfoliated graphene dispersions were characterized by complementary techniques including AFM, DLS, TGA and Raman. Furthermore, treatment of stable dispersions of exfoliated graphene sheets in NMP with poly[styrene-b-(2-vinylpyridine)] block copolymer, under acidic conditions, resulted on aqueous solubilization of graphene. Similar results were obtained, i.e. transfer of graphene from the organic to the aqueous phase, when poly(isoprene-b-acrylic acid) block copolymer was 15 added on exfoliated graphene in NMP.
\end{abstract}

\section{Introduction}

Graphene with a 2D crystal lattice is a unique electronic system possessing exceptional properties ${ }^{1,2}$ suitable for applications in diverse research fields. ${ }^{3-10}$ However, its poor processability, 20 mainly due to insufficient exfoliation from graphite, in combination with its insolubility in all organic solvents, handicap graphene's immediate applicability. Therefore, production of mono- or few-layered graphene is a major challenge and several methods have been developed toward this direction.

25 Micromechanical exfoliation of graphite or the "Scotch tape" method $^{9}$ is widely used to obtain intact graphene layers with perfect structure. However, this method produces low quantities of uneven graphene sheets. Chemical vapour deposition (CVD) of hydrocarbons and epitaxial growth of graphene sheets on metal 30 substrates $^{11-15}$ is another general method for the preparation of graphene in relatively bulk quantities, though the CVD method suffers from limitations in manipulating the size, shape and doping of the as-prepared graphene sheets.

A different approach for obtaining graphene sheets in bulk ${ }_{35}$ quantities is via chemical oxidation of graphite and subsequent exfoliation in water forming soluble graphene oxide. ${ }^{16-21}$ However, in the latter material a variety of oxygen functionalities are introduced, disrupting the extended $\pi$-conjugated $\mathrm{sp}^{2}$ network and affecting the novel electronic properties of graphene, thus 40 making it an insulator. Although reduction of graphene oxide yields reduced graphene oxide sheets, most of the times the process is incomplete and the physical properties of the material produced never reach those of pristine graphene. ${ }^{20-22}$

Recently, another top down method was developed to produce ${ }_{45}$ graphene sheets, involving the exfoliation of graphite in liquid phase, through direct sonication, by using molecules that overcome the strong Van der Waals interaction, supporting the multiple graphene layers in graphite. In this context, several liquids were used to create stable dispersions of graphene such as 50 surfactants in aqueous media, ${ }^{23,24}$ ionic liquids ${ }^{25,26}$ and organic solvents. $^{27-29}$

Moreover, graphene-polymer composites attracted considerable interest because of their great mechanical, electric and thermal properties. ${ }^{31,32}$ Dendritic type polymers were used to 55 functionalize graphene sheets through $\pi-\pi$ stacking, followed by deposition of in-situ generated metal nanoparticles, ${ }^{32}$ while poly(N,N-dimethylacrylamide)-b-poly(N-isopropylacrylamide) block copolymer was intercalated with chemically converted graphene sheets giving rise to a potentially biocompatible hybrid 60 complex. ${ }^{33}$ In a similar way, both hydrophilic and hydrophobic polymers were used to enhance the exfoliation of graphene sheets from graphite in liquid phase through hydrophobic interactions. ${ }^{34-}$ 35 It is also worth-mentioning that aqueous dispersions of graphene can be obtained by sonicating graphite in the presence 65 of polymer as stabilizers such as polyvinyl pyrrolidone or surfactants such as sodium cholate and sodium dodecyl benzene sulfonate. ${ }^{36-42}$

The aim of the current study is the search for new and more efficient strategies for graphene exfoliation and solubilisation not 70 only in organic solvents but also in aqueous media. Herein, the powerful approach of tip sonication was applied to exfoliate graphite and obtain high concentrations of graphene dispersed in organic solvents. Time variation of tip sonication, in parallel with a number of solvents tested was investigated, searching for 75 optimum conditions to achieve maximum exfoliation, while forming stable graphene dispersions. In addition, it is shown that 
the wettability of graphene can be modulated from the organic to the aqueous phase, by adding the amphiphilic block copolymers poly[styrene-b-(2-vinylpyridine)] (abbreviated as PS-b-P2VP) and poly(isoprene-b-acrylic acid) (abbreviated as PI-b-PAA), 5 shown in Scheme 1, as a function of $\mathrm{pH}$.
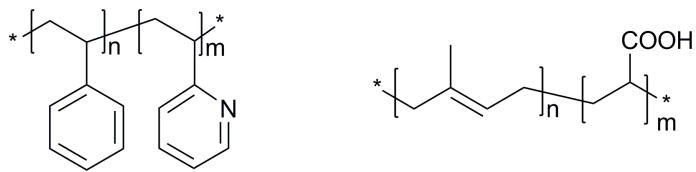

Scheme 1. Chemical structures of PS-b-P2VP (left) and PI-b-PAA (right).

\section{Experimental}

\section{Instrumentation}

10 Tip sonication was performed with a Bandelin Sonoplus Ultrasonic Homogenizer HD 3200 equipped with a flat head probe (VS70T), running at $10 \%$ of the maximum power $(250 \mathrm{~W})$. Centrifugation was performed by an Eppendorf 5702 at 2500 rpm. UV-Vis-IR electronic absorption spectra were recorded on a 15 Perkin-Elmer (Lambda 19) UV-Vis-NIR spectrophotometer. Thermogravimetric analysis was performed using a TGA Q500 V20.2 Build 27 instrument by TA in a nitrogen inert atmosphere. Raman scattering measurements were performed in the backscattering geometry using a RENISHAW inVia Raman

20 microscope equipped with a CCD camera and a Leica microscope at room temperature. A 2400 lines $\mathrm{mm}^{-1}$ grating was used for all measurements, providing a spectral resolution of $\pm 1 \mathrm{~cm}^{-1}$. As an excitation source the $\mathrm{Ar}^{+}$laser $(514 \mathrm{~nm}$ with less than $0.5 \mathrm{~mW}$ laser power) was used. Measurements were taken with $60 \mathrm{~s}$ of 25 exposure times at varying numbers of accumulations. The laser spot was focused on the sample surface using a long working distance 50x objective. Raman spectra were collected on numerous spots on the sample and recorded with a Peltier cooled CCD camera. The intensity ratio $\mathrm{I}_{\mathrm{D}} / \mathrm{I}_{\mathrm{G}}$ was obtained by taking the ${ }_{30}$ peak intensities following any baseline corrections. The data were collected and analysed with Renishaw Wire and Origin software. Dynamic light scattering (DLS) measurements were performed on a ALV/CGS-3 Compact Goniometer System (ALV GmbH, Germany), equipped with a JDS Uniphase $22 \mathrm{~mW} \mathrm{He}-\mathrm{Ne}$ laser, 35 operating at $632.8 \mathrm{~nm}$, interfaced with a ALV-5000/EPP multitau digital correlator with 288 channels and a ALV/LSE-5003 light scattering electronics unit for stepper motor drive and limit switch control. The scattering intensity and correlation functions were measured at $90^{\circ}$. Correlation functions were collected for 40 ten times and were analysed by the cumulant method and the CONTIN software, which provides the apparent hydrodynamic radii distributions by Laplace inversion of the correlation function and by aid of the Stokes-Einstein relationship. Atomic force microscopy (AFM) images were recorded with a Nanoscope IIIa ${ }_{45}$ Extended Multimode (Veeco, USA) in tapping-mode at room temperature under ambient conditions using commercial available etched Si cantilevers with spring constant of $40 \mathrm{nN} \mathrm{m}^{-1}$ and typical frequencies between 200 and $400 \mathrm{kHz}$. All samples for the AFM measurements were prepared by drop casting.

${ }_{50}$ Generally, $5 \mu \mathrm{l}$ solution containing the carbon allotrope was dropped onto freshly cleaved mica wafer with a diameter of 10 $\mathrm{mm}$, and then the mica wafer was placed at an angle of ca. $60^{\circ}$ to let the solution drop flow down at the end of the wafer. Finally, the mica wafer was dried in ambient environment.

\section{${ }_{55}$ Materials and Reagents}

Graphite flakes (75+ mesh, $>75 \%$, batch No: 13802EH) and all solvents were purchased from Aldrich and SDS and used as received without further purification. Poly[styrene-b-(2vinylpyridine)] PS-b-P2VP block copolymer $\left(\mathrm{M}_{\mathrm{w}}=115,000\right.$, ${ }_{60} \mathrm{M}_{\mathrm{w}} / \mathrm{M}_{\mathrm{n}}=1.02,44 \%$ PS) and poly(isoprene-b-acrylic acid) PI-bPAA $\left(\mathrm{M}_{\mathrm{w}}=42500, \mathrm{M}_{\mathrm{w}} / \mathrm{M}_{\mathrm{n}}=1.16,10 \% \mathrm{wt}\right.$ PI $)$ were synthesized following known procedures. ${ }^{43,44}$

\section{Graphene exfoliation in organic solvents}

In a typical experiment, $50 \mathrm{mg}$ of graphite flakes were added in ${ }_{65} 100 \mathrm{~mL}$ of the examined solvent, [N,N-dimethylformamide (DMF), tetrahydrofuran (THF), dimethyl sulfoxide (DMSO), pyridine, o-dichlorobenzene (o-DCB) and N-methyl-1,2pyrolidone (NMP)]. The mixture was sonicated for various times $(5,15,30$ and 60 minutes). The obtained ink-like graphene 70 dispersion was centrifuged for $15 \mathrm{~min}$ and the supernatant was collected and analyzed.

\section{Graphene/amphiphilic block copolymers ensembles in water}

A stock solution of amphiphilic block copolymers PS-b-P2VP $(0.6 \mathrm{mg} / \mathrm{mL})$ and PI-b-PAA $(0.5 \mathrm{mg} / \mathrm{mL})$ in NMP was prepared 75 and added to exfoliated graphene dispersions in NMP at a weight ratio $2: 1,1: 1$ and $0.5: 1$, respectively (see below for details, Table $\mathrm{S} 1$, entry 4 ). Then, $1 \mathrm{~mL}$ of the graphene/PS-b-P2VP ensemble was added to $5 \mathrm{~mL}$ of vigorously stirred $\mathrm{HCl}$ aqueous solution $(\mathrm{pH} \approx 2)$ and $1 \mathrm{~mL}$ of the graphene/PI-b-PAA ensemble was added 80 to $5 \mathrm{~mL}$ of vigorously stirred $\mathrm{HCl}$ aqueous solution $(\mathrm{pH} \approx 2)$, distilled $\mathrm{H}_{2} \mathrm{O}(\mathrm{pH} \approx 7), \mathrm{Na}_{2} \mathrm{CO}_{3}$ aqueous solution $(\mathrm{pH} \approx 9)$, and $\mathrm{NaOH}$ aqueous solution ( $\mathrm{pH} \approx 12$ ), respectively.

\section{Results and discussion}

According to the procedures described in the experimental 85 section, the optimum conditions to achieve efficient graphene exfoliation from commercially available graphite flakes was tip sonication at 25 Watt for $60 \mathrm{~min}$. Actually, among all solvents tested (i.e. DMF, THF, DMSO, pyridine, o-DCB and NMP) the most stable dispersions and with the most intense colour, as a first 90 hint for the success of the protocol followed, were obtained in $\mathrm{NMP}^{27}$ and o-DCB. ${ }^{29}$ Dark grey to black coloured dispersion of exfoliated graphene were obtained after centrifugation, in sharp contrast with the colourless dispersions formed, just like the solvent colour before sonication of graphite flakes, in the rest of 95 the examined solvents. At this stage, it is concluded that o-DCB and NMP are ideal solvents for the exfoliation of graphite, with the o-DCB being best. In Fig. 1, digital images of exfoliated graphene obtained after $5,15,30$ and 60 minutes of tip sonication in o-DCB as compared to pristine graphite are shown. Evidently, 100 pristine graphite is completely insoluble, while the shortsonicated sample (i.e. $5 \mathrm{~min}$.) in o-DCB was found grey and the solution colouration gradually turned black as the sonication time increased. Obviously, the highest concentration of dispersed graphene was achieved after $60 \mathrm{~min}$. of tip sonication in o-DCB. 105 At this point it should be emphasized that o-DCB is decomposed 


\section{Cite this: DOI: $10.1039 / \mathrm{c0xx} 00000 x$}

ARTICLE TYPE

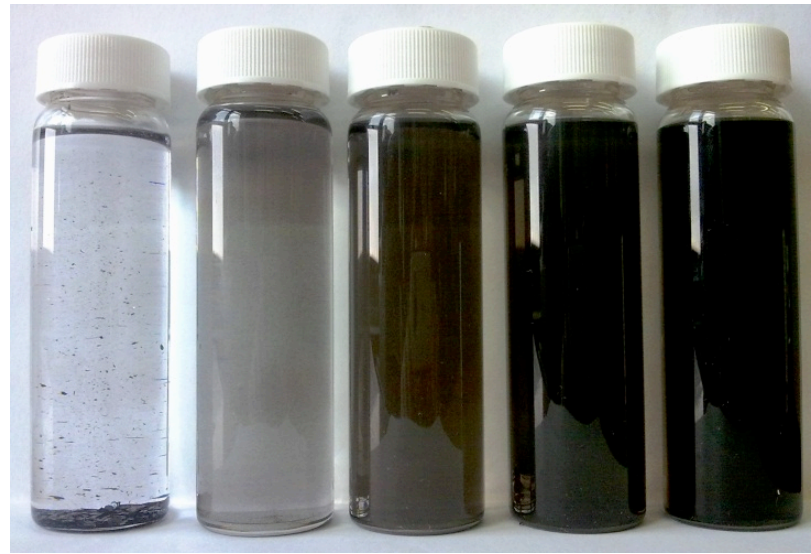

Fig. 1. From left to right: pristine graphite and exfoliated graphene as obtained by tip sonication for 5, 15, 30 and 60 minutes, in o-DCB.

upon sonication, however, the small impurities generated may result on additional stabilization of the exfoliated graphene. ${ }^{45-47}$ ${ }_{5}$ When a blank experiment in which o-DCB was tip sonicated, under the same experimental conditions followed for the graphene exfoliation, the generation of a small amount of decomposed products, mainly precipitated upon centrifugation, was similarly found.

10 For the estimation of solubility values for exfoliated graphene, UV-VIS spectroscopy was used (see experimental details in the ESI $\dagger$ ). Initially, the molar absorptivity of exfoliated graphene was calculated as $3,780 \mathrm{~L} \mathrm{~g}^{-1} \mathrm{~m}^{-1}$ at $660 \mathrm{~nm}$ (Fig. S1, ESI $\dagger$ ) and then by applying the Beer-Lambert law with the aid of a titration 15 curve, the solubility values in NMP and o-DCB were calculated (Table S1, ESI $\dagger$ ). Based on those measurements and calculations, the concentration of exfoliated graphene after $60 \mathrm{~min}$. of tip sonication in o-DCB was found $17.8 \mu \mathrm{g} / \mathrm{mL}$, whilst at the same time the exfoliated graphene dispersion in NMP was measured at ${ }_{20} 3.8 \mu \mathrm{g} / \mathrm{mL}$.

Raman spectroscopy is a powerful tool for characterizing carbon nanostructures. In Fig. 2, the Raman spectrum of exfoliated graphene is shown. A characteristic band at $1581 \mathrm{~cm}^{-1}$, (G-band, $\mathrm{sp}^{2}$ carbons), upshifted by ca. $1 \mathrm{~cm}^{-1}$ as compared with 25 that of intact graphite is observed. In addition, a D-band at 1350 $\mathrm{cm}^{-1}$ (which is absent in pristine graphite) and the 2D-band at $2693 \mathrm{~cm}^{-1}$ (Fig. 2) are evident. The presence of a D-band with a relatively high intensity $\left(\mathrm{I}_{\mathrm{D}} / \mathrm{I}_{\mathrm{G}}=0.6\right)$ in exfoliated graphene is due to $\mathrm{sp}^{3}$ hybridized carbon atoms and structural defects caused by 30 the tip sonication applied to achieve exfoliation from graphite. On the other hand, the shape and frequency of 2D-band is sensitive to the number of graphene layers, namely it becomes smoother and broader upon exfoliation of graphite to oligolayered graphene sheets, while it sharpens for monolayered graphene. ${ }^{12,14}$ ${ }_{35}$ Evidently, the 2D-band of exfoliated graphene appears at 2693 $\mathrm{cm}^{-1}$ broader and downshifted by ca. $32 \mathrm{~cm}^{-1}$ as compared with that of intact graphite, thus, unambiguously suggesting the presence of few-layered graphene.

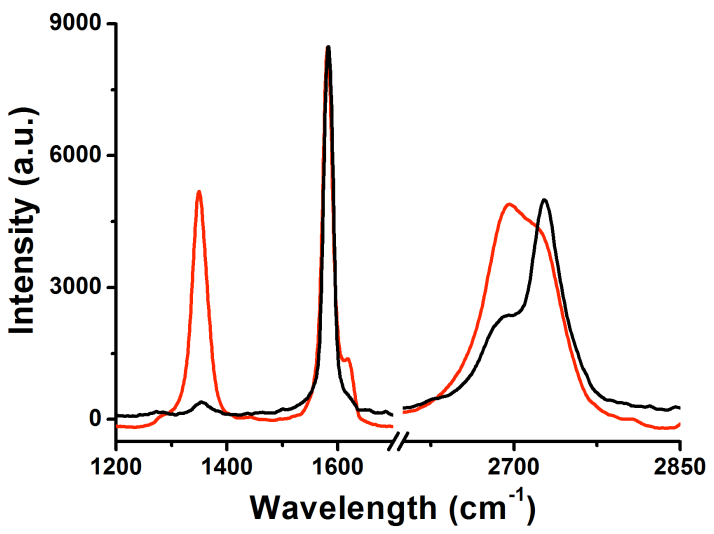

40 Fig. 2. Raman spectra of pristine graphite flakes (black) and exfoliated graphene (red) as obtained by tip sonication for 60 minutes in o-DCB $\left(\lambda_{\text {exc. }}=514 \mathrm{~nm}\right)$.

DLS measurements confirmed the presence of structural changes on exfoliated graphene caused by tip sonication. The 45 apparent hydrodynamic radius $\left(\mathrm{R}_{\mathrm{h}}\right)$ of exfoliated graphene sheets, estimated using cumulant analysis, was found ca. $200 \mathrm{~nm}$, suggesting that graphene sheets are small-sized.

Morphological examination of exfoliated graphene was performed by AFM. Exfoliated graphene in either o-DCB or 50 NMP was deposited onto fresh cleaved mica substrate, then dried in air and the height and topography of the so-prepared exfoliated graphene films were studied. Because of their high surface area, graphene sheets were aggregated, tend to coalesce and overlap each other forming wrinkled structures or even re-stacked to a ${ }_{55}$ graphitic structure due to van der Waals interactions when their dispersion was dried. A typical AFM image of graphene sheets is presented in Fig. 3. Profile analysis shows a height of $2.5 \mathrm{~nm}$ for the region indicated by arrows. Section analysis of other regions of the image show height ranges between $1.5 \mathrm{~nm}$ and $20 \mathrm{~nm}$ and 60 lateral dimensions of $40-50 \mathrm{~nm}$, indicating the presence of smallsized oligolayered graphene sheets.

In the next step graphene/copolymer ensembles in organic solvent (i.e. NMP) were examined by DLS measurements. After PS-b-P2VP block copolymer addition to exfoliated graphene ${ }_{65}$ dispersions in NMP, no significant changes to the average apparent hydrodynamic radius of the dispersed nanostructures were observed. On the other hand a noticeable decrease was observed in this parameter when PI-b-PAA was added to the graphene dispersions in NMP. 


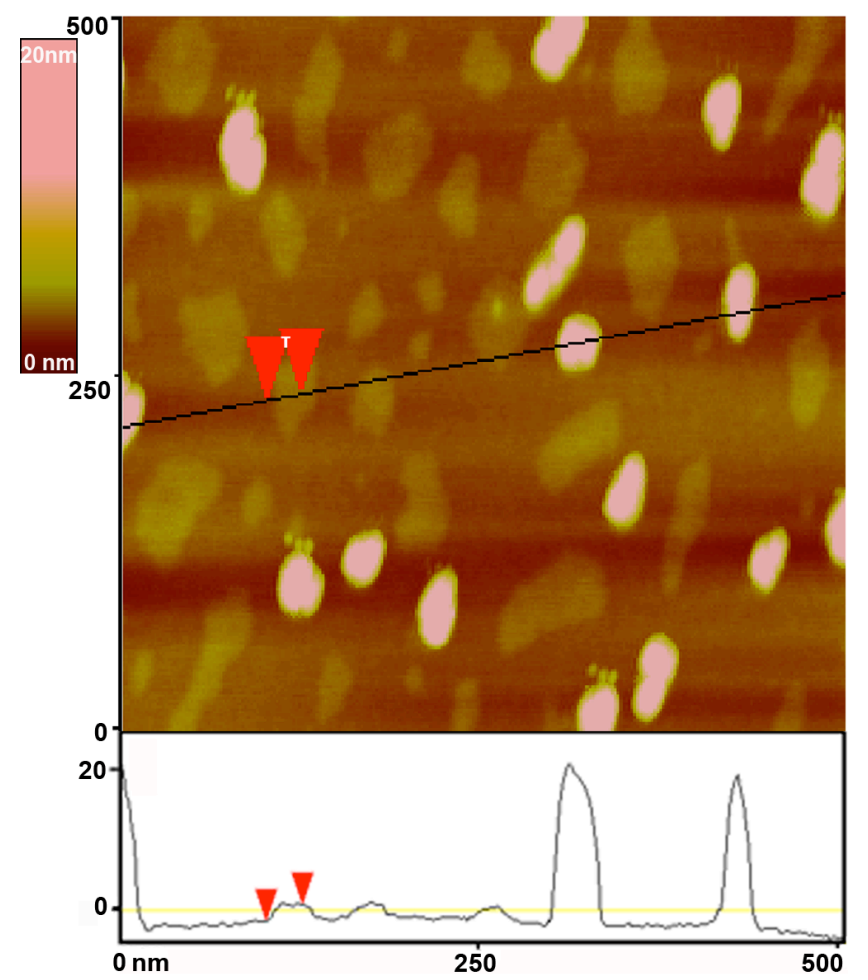

Fig. 3. AFM Image and section analysis of exfoliated graphene sheets dispersed in NMP (height difference between arrows is $2.5 \mathrm{~nm}$ ).

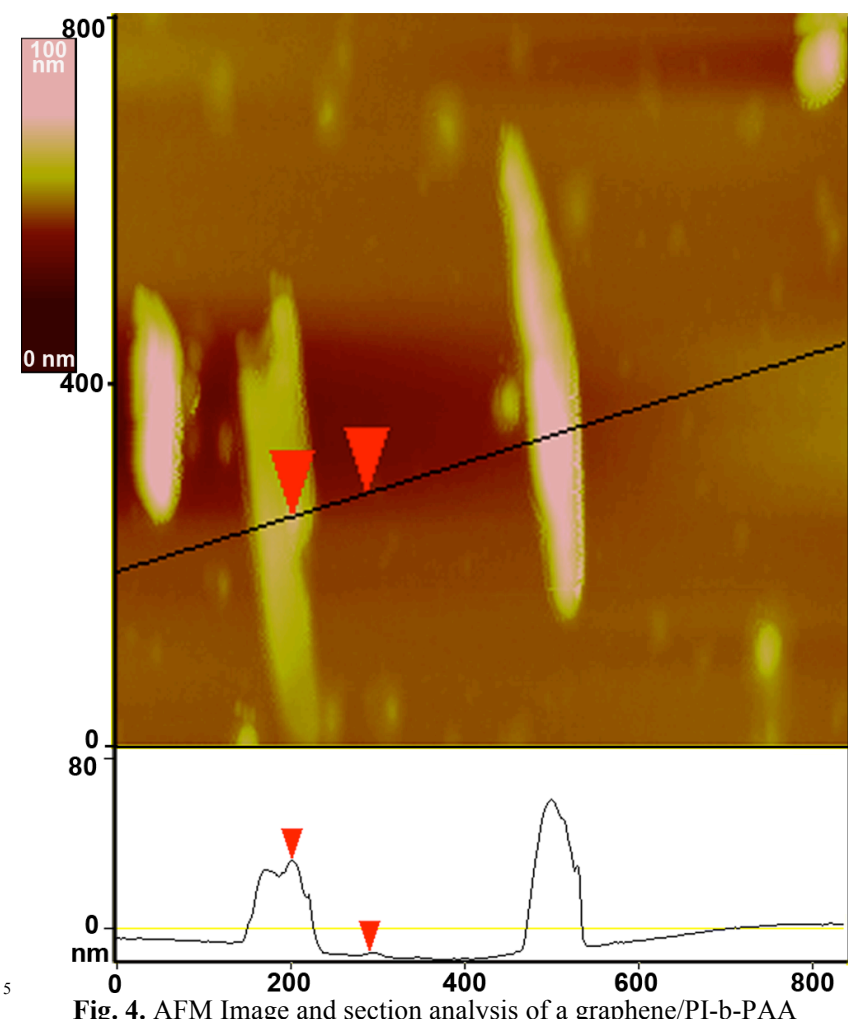

Fig. 4. AFM Image and section analysis of a graphene/PI-b-PAA ensemble dispersed in NMP (height difference between arrows is $44 \mathrm{~nm}$ ).

AFM imaging was utilized in order to extract more detailed information on the block copolymer/graphene hybrid 10 nanostructures. Images obtained indicate that the morphology of graphene/PI-b-PAA ensembles (Fig. 4) is quite different compared with that of exfoliated graphene (cf. Fig. 3). The presence of amphiphilic PI-b-PAA block copolymer in the exfoliated graphene dispersions resulted to the formation of 15 heterogeneous sized rod-like shaped aggregations, with thickness ranging from $35 \mathrm{~nm}$ to $70 \mathrm{~nm}$ and lateral dimensions around 450 $\mathrm{nm}$. Profile analysis shows a $44 \mathrm{~nm}$ height for the indicated by arrows section (Fig. 4). Although there seems to be a disagreement with the DLS results one should keep in mind that 20 the results of the two methods are not directly comparable. DLS gives an apparent average hydrodynamic radius that depends on size distribution and shape of the dispersed nanostructure. AFM probes the nanostructures in the solvent free state on a solid surface. Correlation of the results in this case may indicate a re25 assembly of the block copolymer/graphene nanoaggregates on the solid substrate. On the other hand, AFM images of graphene/PSb-P2VP ensembles (Fig. S2, ESI $\dagger$ ) show the presence of uniformly shaped and sized sheets, with thickness between 20-30 $\mathrm{nm}$ and lateral dimension of $50 \mathrm{~nm}$, quite similar to exfoliated 30 graphene sheets.

The approximate weight fraction of the physically adhered block copolymer on exfoliated graphene was determined by thermogravimetric analysis. TGA measurements performed under inert nitrogen atmosphere, so intact graphite flakes are thermally 35 stable up to $900{ }^{\circ} \mathrm{C}$, contrary to exfoliated graphene which showed a continuous weight loss most likely due to the incorporation of defects during tip sonication (Fig. 5). Graphene/PS-b-P2VP hybrid material showed a total weight loss of $40 \%$ up to $550{ }^{\circ} \mathrm{C}$ (Fig. 5a) which can be attributed to 40 decomposition of the organic addends grafted onto exfoliated graphene sheets, including the copolymer moieties. Intact PS-bP2VP decomposed at $405{ }^{\circ} \mathrm{C}$, under nitrogen atmosphere. Likewise, graphene/PI-b-PAA hybrid material showed a two-step weight loss of $55 \%$ up to $550{ }^{\circ} \mathrm{C}$ (Fig. 5b). In both cases, the 45 observed weight loss above $550{ }^{\circ} \mathrm{C}$ is attributed to the destruction of the graphitic skeleton starting from defects sites. 

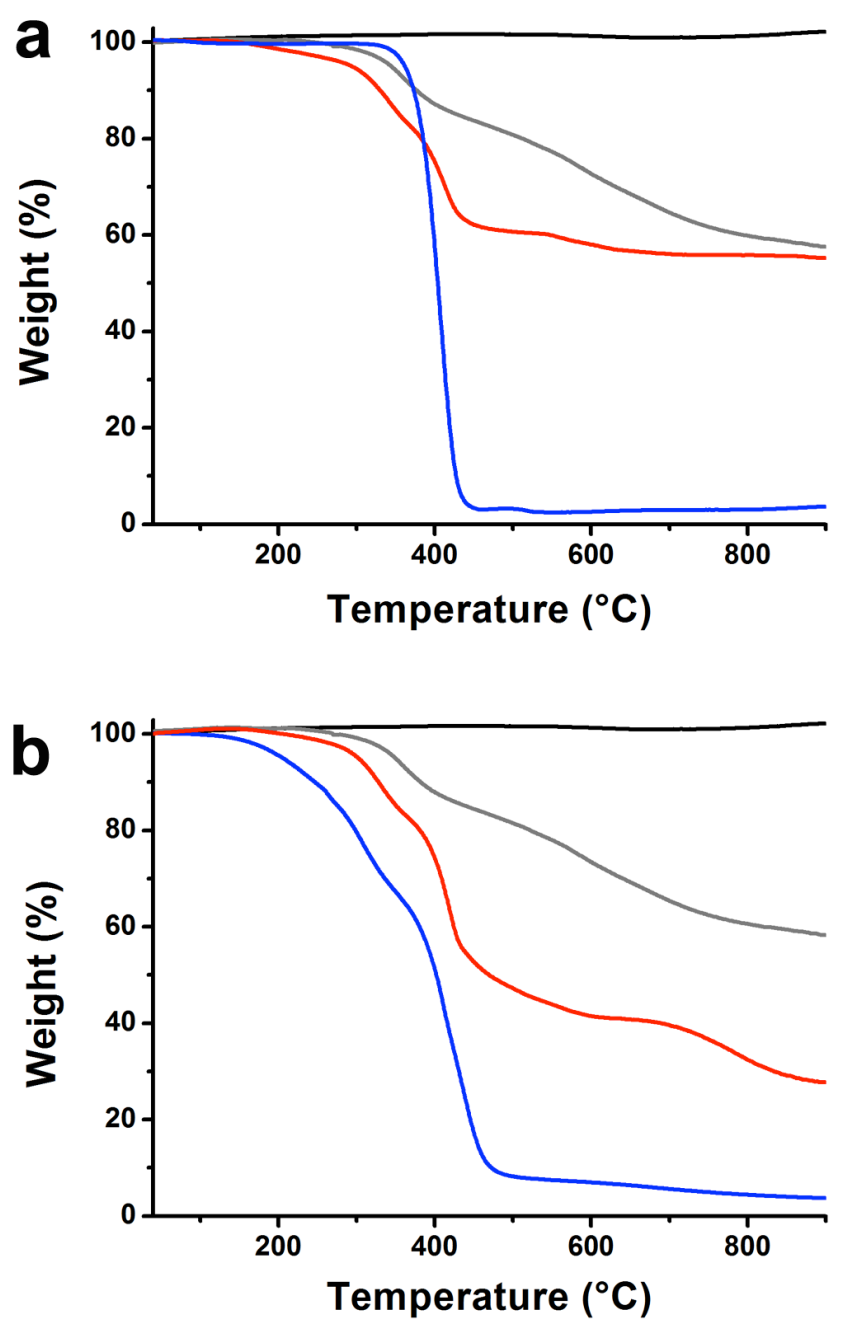

Fig. 5. Thermographs of intact graphite flakes (black), exfoliated graphene as obtained by tip sonication for 60 minutes in NMP (gray) together with a) graphene/PS-b-P2VP (red) and PS-b-P2VP copolymer

5 (blue), and b) graphene/PI-b-PAA (red) and PI-b-PAA copolymer (blue).

Having achieved the exfoliation of graphene in organic solvents, we turned our efforts on switching its solubility from the organic phase to an aqueous environment. Because o-DCB miscibility with water is almost negligible, while NMP shows 10 better affinity with water, the studies for transferring the exfoliated graphene sheets to water were performed in the latter organic solvent. Briefly, three different graphene/PS-b-P2VP ensembles with weight ratios adjusted to $1: 2,1: 1$ and 1:0.5 were prepared by adding $0.18,0.35$ and $0.7 \mathrm{mg}$ of PS-b-P2VP into 15 exfoliated graphene in NMP $(30 \mathrm{~mL})$. Then, $1 \mathrm{~mL}$ of each dispersion was added dropwise into $5 \mathrm{~mL}$ of vigorously stirring $\mathrm{HCl}$ aqueous solution $(\mathrm{pH} \approx 2)$. It is important to adjust the $\mathrm{pH}$ in an acidic value, in order to protonate the pyridine group of PS-bP2VP and promote its water solubility. Actually, the positive
20 charges on the copolymers are responsible for the aqueous solubilization of the graphene/PS-b-P2VP ensemble in an acidic environment, as it was observed in Fig. 6.

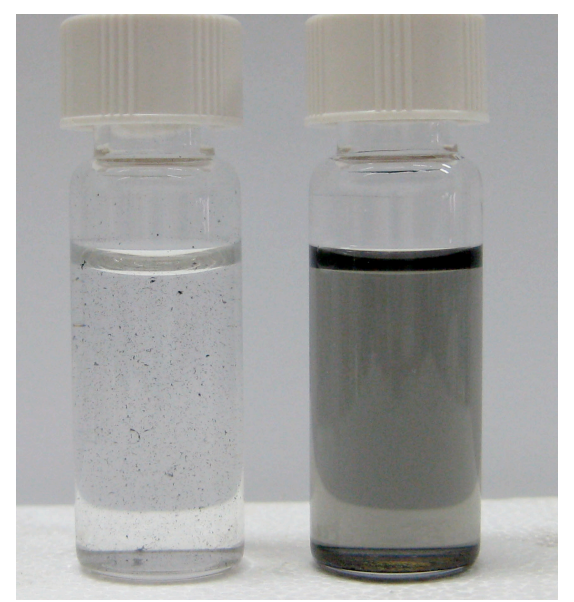

Fig. 6 Graphene/PS-b-P2VP in 1:5 vol/vol NMP: $\mathrm{H}_{2} \mathrm{O}$, at $\mathrm{pH}=7$ (left vial) 25 and at $\mathrm{pH}=2$ (right vial).

For the case of the amphiphilic block copolymer PI-b-PAA, a similar procedure was followed and the graphene/ PI-b-PAA ensemble was transferred into water. At this point it should be emphasized that PI-b-PAA block copolymer possesses $-\mathrm{COOH}$ 30 functions sensitive to $\mathrm{pH}$ changes. Deprotonation of the carboxylic unit occurs at $\mathrm{pH}$ values above 4 , and this is where substantial aqueous solubilisation was observed. However, as poly(acrylic acid) is water soluble even at acidic $\mathrm{pH}$ aqueous solubility of PI-b-PAA and therefore of the graphene/PI-b-PAA 35 ensemble is expected to be $\mathrm{pH}$ insensitive.

The aqueous phase solutions that were prepared by adding the organic solutions containing graphene and PS-b-P2VP into acidified water $(\mathrm{pH} \approx 2)$ were characterized by DLS. In this case protonation of the pyridine moiety of the graphene/PS-b-P2VP 40 nanoassemblies is expected. Regardless the graphene/copolymer weight fraction in aqueous dispersions there is a relative aggregation of the initial nanoentities as evidenced by the increase of $R_{h}$ from ca. $200 \mathrm{~nm}$ to ca. $500 \mathrm{~nm}$. Aggregation effects also occurred in the graphene/PI-b-PAA dispersions in 45 neutral and acidic aqueous media. Apparent $\mathrm{R}_{\mathrm{h}}$ increased to ca. $800-1100 \mathrm{~nm}$ in neutral $\mathrm{pH}$ and to $600 \mathrm{~nm}$ in acidic environment. In basic conditions the apparent $\mathrm{R}_{\mathrm{h}}$ increased to ca. $700 \mathrm{~nm}$ at $\mathrm{ph} \approx 9$, while at $\mathrm{pH} \approx 12$ increased to ca. $900 \mathrm{~nm}$. The carboxyl groups of the PAA blocks of the copolymer deprotonate at $\mathrm{pH}>4$, 50 so an increase in $\mathrm{R}_{\mathrm{h}}$ was expected as $\mathrm{pH}$ was decreased, due to a decrease in the solvent quality, which in turn would result in aggregation of the hybrid nanoassemblies. At basic $\mathrm{pH}$ (ca. 9 and 12) an increase of $R_{h}$ is also observed but since here deprotonation of the $\mathrm{COOH}$ moieties of the graphene/PI-b-PAA ${ }_{55}$ nanoassemblies is expected, the increase in dimensions can be 
also explained by the tendency of PI-b-PAA adsorbed chains to extend their molecular dimensions in basic environment. In any case the hydrophobicity of graphene moieties present in the hybrid nanoassemblies is expected to induce aggregation of the 5 initial nanostructures when solvent is changed to water. The aqueous dispersions were monitored monthly to verify their stability. No significant precipitation of the large graphene flakes was observed for the first five months with a small but not significant decrease in apparent $R_{h}$.

10 Finally, AFM imaging of the aqueous dispersions of graphene/PS-b-P2VP hybrid material (Fig. 7) confirmed the existence of single- and few-layered graphene sheets. Graphene/PS-b-P2VP ensembles are not homogeneously sized, however, section analysis showed that the observed 15 graphene/copolymer ensembles are only $1 \mathrm{~nm}$ to $7 \mathrm{~nm}$ thick.

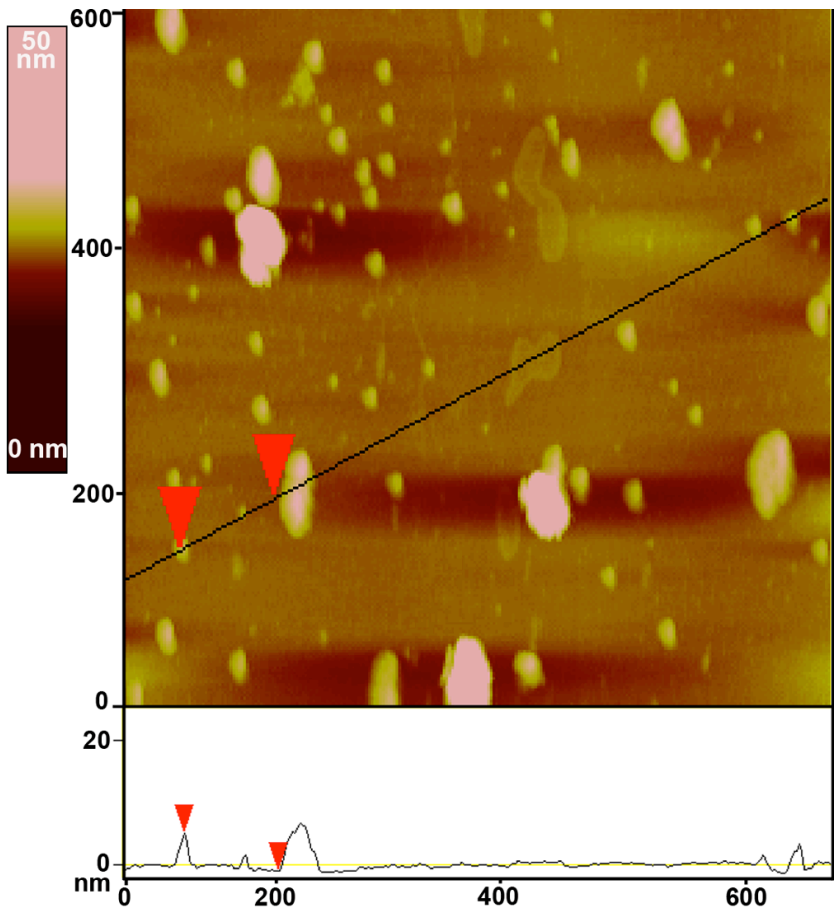

Fig. 7. AFM image and section analysis of a graphene/PS-b-P2VP ensemble dispersed in water (height difference between arrows is $6 \mathrm{~nm}$ ).

\section{Conclusions}

${ }_{20}$ Graphene sheets were exfoliated upon tip sonication in a variety of organic solvents. It was found that stable graphene dispersions could be formed in o-DCB and NMP. Spectroscopic, thermal and microscopy techniques were applied to characterize the exfoliated graphene sheets. Furthermore, when exfoliated graphene in NMP 25 was treated with an acidic solution of poly[styrene-b-(2vinylpyridine)] block copolymer, aqueous solubilization of graphene was achieved. In addition, when exfoliated graphene was treated with poly(isoprene-b-acrylic acid) block copolymer the dispersibility of exfoliated graphene was switched from the ${ }_{30}$ organic to the aqueous phase.

\section{Acknowledgements}

Partial financial support from GSRT/NSRF 2007-2013

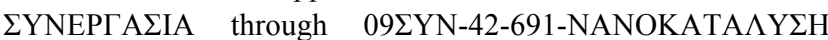

Project, the action "Postdoctoral support" Project GRAPHCELL ${ }_{35}$ PE5(2126) and the Program "Visiting Professorship for Senior International Scientists" by the Chinese Academy of Sciences to N.T. is acknowledged.

\section{Notes and References}

${ }^{a}$ Theoretical and Physical Chemistry Institute, National Hellenic

40 Research Foundation, 48 Vassileos Constantinou Avenue, Athens 11635, Greece.E-mail: tagmatar@eie.gr (N.Tagmatarchis); pispas@eie.gr (S. Pispas).

${ }^{b}$ Institute of Chemistry, Chinese Academy of Sciences, Beijing 100190, China.

$45 \dagger$ Electronic Supplementary Information (ESI) available: Procedure for calculating solubility values of exfoliated graphene, titration curve for the estimation of absorption coefficient of exfoliated graphene, solubility values of exfoliated graphene and AFM image and section analysis of graphene/PS-b-P2VP. See DOI: 10.1039/b000000x/

501 R. R. Nair, P. Blake, A. N. Grigorenko, K. S. Novoselov, T. J. Booth, T. Stauber, N. M. R. Peres and A. K. Geim, Science, 2008, 320, 1308.

2 C. Lee, X. Wei, J. W. Kysar and J. Hone, Science, 2008, 321, 385.

3 K. S. Novoselov, A. K. Geim, S. V. Morozov, D. Jiang, Y. Zhang, S.

55 V. Dubonos, I. V. Grigorieva and A. A. Firsov, Science, 2004, 306, 666.

4 X. Wang, L. Zhi and K. Mullen, NanoLett., 2007, 8, 323.

5 Y.-M. Lin, C. Dimitrakopoulos, K. A. Jenkins, D. B. Farmer, H.-Y. Chiu, A. Grill and P. Avouris, Science, 2010, 327, 662.

${ }_{60} 6$ S. Stankovich, D. A. Dikin, G. H. B. Dommett, K. M. Kohlhaas, E. J. Zimney, E. A. Stach, R. D. Piner, S. T. Nguyen and R. S. Ruoff, Nature, 2006, 442, 282.

7 L. Qu, Y. Liu, J.-B. Baek and L. Dai, ACS Nano, 2010, 4, 1321-1326.

8 Y. Liu, D. Yu, C. Zeng, Z. Miao and L. Dai, Langmuir, 2010, 26, 6158.

9 A. K. Geim and K. S. Novoselov, Nat. Mater., 2007, 6, 183.

10 H. Chen, M. B. Müller, K. J. Gilmore, G. G. Wallace and D. Li, $A d v$. Mater., 2008, 20, 3557.

11 P. W. Sutter, J.-I. Flege and E. A. Sutter, Nat Mater, 2008, 7, 406.

7012 A. Reina, X. Jia, J. Ho, D. Nezich, H. Son, V. Bulovic, M. S. Dresselhaus and J. Kong, NanoLett., 2009, 9, 30.

13 K. V. Emtsev, A. Bostwick, K. Horn, J. Jobst, G. L. Kellogg, L. Ley, J. L. McChesney, T. Ohta, S. A. Reshanov, J. Rohrl, E. Rotenberg, A. K. Schmid, D. Waldmann, H. B. Weber and T. Seyller, Nat. Mater., 2009, 8, 203.

14 K. S. Kim, Y. Zhao, H. Jang, S. Y. Lee, J. M. Kim, K. S. Kim, J.-H. Ahn, P. Kim, J.-Y. Choi and B. H. Hong, Nature, 2009, 457, 706.

15 X. Li, W. Cai, J. An, S. Kim, J. Nah, D. Yang, R. Piner, A. Velamakanni, I. Jung, E. Tutuc, S. K. Banerjee, L. Colombo and R. S. Ruoff, Science, 2009, 324, 1312.

16 S. Stankovich, D. A. Dikin, R. D. Piner, K. A. Kohlhaas, A. Kleinhammes, Y. Jia, Y. Wu, S. T. Nguyen and R. S. Ruoff, Carbon, $2007, \mathbf{4 5}, 1558$

17 D. Li, M. B. Muller, S. Gilje, R. B. Kaner and G. G. Wallace, Nat. Nanotechnol., 2008, 3, 101.

18 S. Park and R. S. Ruoff, Nat. Nanotechnol., 2009, 4, 217-224

19 D. R. Dreyer, S. Park, C. W. Bielawski and R. S. Ruoff, Chem. Soc. Rev., 2010, 39, 228.

20 S. Stankovich, R. D. Piner, S. T. Nguyen and R. S. Ruoff, Carbon, 2006, 44, 3342

21 R. Muszynski, B. Seger and P. V. Kamat, J. Phys. Chem. C, 2008, 112, 5263.

22 K. Tanaka and F. Toda, Chem. Rev., 2000, 100, 1025.

23 M. Lotya, Y. Hernandez, P. J. King, R. J. Smith, V. Nicolosi, L. S. Karlsson, F. M. Blighe, S. De, Z. Wang, I. T. McGovern, G. S. Duesberg and J. N. Coleman, J. Am. Chem. Soc., 2009, 131, 3611.

24 M. Lotya, P. J. King, U. Khan, S. De and J. N. Coleman, ACS Nano, $2010,4,3155$.

25 B. Zhang, W. Ning, J. Zhang, X. Qiao, J. Zhang, J. He and C.-Y. Liu, 100 J. Mater. Chem., 2010, 20, 5401. 
26 D. Nuvoli, L. Valentini, V. Alzari, S. Scognamillo, S. B. Bon, M. Piccinini, J. Illescas and A. Mariani, J. Mater. Chem., 2011, 21, 3428.

27 Y. Hernandez, V. Nicolosi, M. Lotya, F. M. Blighe, Z. Sun, S. De, I. T. McGovern, B. Holland, M. Byrne, Y. K. Gun'Ko, J. J. Boland, P. Niraj, G. Duesberg, S. Krishnamurthy, R. Goodhue, J. Hutchison, V. Scardaci, A. C. Ferrari and J. N. Coleman, Nat. Nanotechnol., 2008, 3, 563.

28 S. P. Economopoulos, G. Rotas, Y. Miyata, H. Shinohara and N. Tagmatarchis, ACS Nano, 2010, 4, 7499

29 C. E. Hamilton, J. R. Lomeda, Z. Sun, J. M. Tour and A. R. Barron, NanoLett., 2009, 9, 3460.

30 H. Bai, C. Li and G. Shi, Adv. Mater., 2011, 23, 1089.

31 H. J. Salavagione, G. Martínez and G. Ellis, Macromol. Rapid Commun., 2011, 32, 1771 .

32 H. Li, L. Han, J. J. Cooper-White and I. Kim, Nanoscale, 2012, 4, 1355.

33 J. Liu, G. Chen and M. Jiang, Macromolecules, 2011, 44, 7682.

34 J. Y. Lee and I. In, Chem. Lett., 2011, 40, 567.

2035 I. Tantis, G. C. Psarras and D. Tasis, Expr. Polym. Lett., 2012, 6, 283

36 O. V. Pupysheva, A. A. Farajian, C. R. Knick, A. Zhamu and B. Z. Jang, J. Phys. Chem. C, 2010, 114, 21083.

37 S. Vadukumpully, J. Paul and S. Valiyaveettil, Carbon, 2009, 47, 3288 .

2538 S. De, P. J. King, M. Lotya, A. O’Neill, E. M. Doherty, Y. Hernandez, G. S. Duesberg and J. N. Coleman, Small, 2010, 6, 458.

39 A. A. Green and M. C. Hersam, NanoLett., 2009, 9, 4031.

40 M. Lotya, Y. Hernandez, P. J. King, R. J. Smith, V. Nicolosi, L. S. Karlsson, F. M. Blighe, S. De, Z. M. Wang, I. T. McGovern, G. S. Duesberg and J. N. Coleman, J. Am. Chem. Soc., 2009, 131, 3611.

41 M. Lotya, P. J. King, U. Khan, S. De and J. N. Coleman, ACS Nano, $2010,4,3155$.

42 R. Hao, W. Qian, L. H. Zhang and Y. L. Hou, Chem. Commun., 2008, 6576 .

3543 D. Topouza, K. Orfanou and S. Pispas, J. Polym. Sci., Part A: Polym. Chem., 2004, 42, 6230.

44 S. Pispas, J. Phys. Chem. B, 2006, 110, 2649.

45 S. Niyogi, M. A. Hamon, D. E. Perea, C. B. Kang, B. Zhao, S. K. Pal, A. E. Wyant, M. E. Itkis, and R. C. Haddon, J. Phys. Chem. B, 2003, 107, 8799 .

46 D. S. Kim, D. Nepal and K. E. Geckeler, Small, 2005, 1, 1117-1124.

47 C. E. Hamilton, J. R. Lomeda, Z. Sun, J. M. Tour and A. R. Barron, Nano Lett., 2009, 9, 3460-3462. 


\section{Table of Contents}

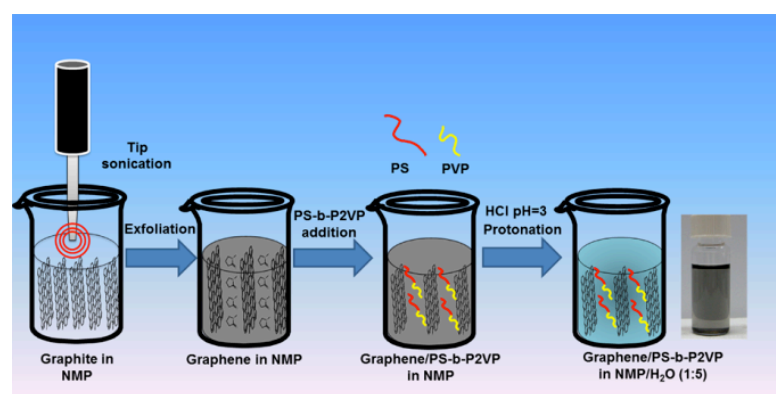

The exfoliation of graphite to graphene sheets in organic solvents via tip sonication and the transfer of graphene from organic to aqueous phase with the aid of amphiphilic block copolymers was achieved. 